\title{
ACMT Position Statement: Interim Guidance for the Use of Lipid Resuscitation Therapy
}

\author{
American College of Medical Toxicology
}

Published online: 17 February 2011

(C) American College of Medical Toxicology 2011

Keywords Lipid resuscitation · Intralipid · ACMT

Lipid resuscitation therapy (LRT) refers to the administration of a lipid emulsion with the intent of reducing the clinical manifestations of toxicity from excessive doses of certain medications. This therapy has shown very promising results in experimental (animal) models of poisoning by lipid-soluble cardiotoxic medications $[1,2]$. The data deriving from experience with similarly poisoned humans is highly anecdotal, although they do suggest that LRT may be beneficial $[1,2]$. The choice of whether, and when, to initiate LRT is one that is solely discretionary and is based on the clinical judgment of the treating physician. Given the uncertainty of its beneficial effect in human poisonings, it is the opinion of the American College of Medical Toxicology (ACMT) that there are no standard of care requirements to use, or to choose not to use, LRT. However, in circumstances where there is serious hemodynamic, or other, instability from a xenobiotic with a high degree of lipid solubility, LRT is viewed as a reasonable consideration for therapy, even if the patient is not in cardiac arrest.

The purpose of this document is to propose a treatment guideline if LRT is used as a component of the treatment of poisoned patients. If LRT is used, it should be instituted for patients with hemodynamic, or other (e.g., intractable seizures), instability, not responsive to standard resuscitation measures, such as fluid replacement, inotropes, and pressors, where appropriate. The decision to use LRT instead of, or in conjunction with, other therapies, such as

American College of Medical Toxicology

10645 N. Tatum Blvd., Suite 200-111,

Phoenix, AZ 85028, USA

e-mail: jmt@acmt.net

euglycemic insulin therapy, is to be based on the clinical judgment of the treating physician. Where possible, it is recommended that these therapies be administered in consultation with a medical toxicologist.

\section{Recommended Guideline}

If the decision is made to initiate LRT, the following guideline is recommended. This suggested guideline is a modification of the ones posted on the LipidRescue.org [1], the UK Resuscitation Council [3], and the Association of Anaesthetists of Great Britain and Ireland [4]. However, it is completely appropriate if the treating physician, based on his/her clinical judgment, chooses to alter the manner in which LRT is administered.

1. Twenty percent lipid emulsion (e.g., Intralipid ${ }^{1}$ ) should be administered as a $1.5-\mathrm{ml} / \mathrm{kg}$ bolus. This can be accomplished by drawing the appropriate volume of $20 \%$ lipid emulsion into $50 \mathrm{ml}$ syringes and administering it through an intravenous catheter. The bolus should be administered over 2-3 min.

2. The bolus should be followed immediately by an infusion of $20 \%$ lipid emulsion at a rate of $0.25 \mathrm{ml} / \mathrm{kg} / \mathrm{min}$. This can be accomplished by attaching the lipid emulsion bag to an intravenous pump. Blood pressure, heart rate, and other available hemodynamic parameters should be recorded at least every $15 \mathrm{~min}$ during the infusion.

\footnotetext{
${ }^{1}$ The proprietary product Intralipid is recommended here because it was the test material in most published studies and utilized in most case reports, where the specific lipid product was specified. It is unknown whether comparable results would have been obtained if lipid products with different composition were utilized.
} 
3. For asystolic patients, or those with pulseless electrical activity, who do not respond to the bolus, the dose may be repeated.

4. If there is an initial response to the bolus followed by the re-emergence of hemodynamic instability, the infusion rate could be increased or, in severe cases, the bolus could be repeated.

5. Where possible, LRT should be terminated after $1 \mathrm{~h}$, or less, if the patient's clinical status permits. In cases where the patient's stability is dependent on continued lipid infusion, longer periods of treatment may be appropriate.

\section{References}

1. Weinberg G (2007) LipidRescue: resuscitation for cardiac toxicity. http://www.lipidrescue.org/
2. Cave G, Harvey M (2009) Intravenous lipid emulsion as antidote beyond local anesthetic toxicity: a systematic review. Acad Emerg Med 16:815-824

3. Resuscitation Council (UK) (2010) Resuscitation Council (UK). http://www.resus.org.uk/

4. AAGBI (2010) The Association of Anaesthetists of Great Britain and Ireland. http://www.aagbi.org/

This guideline has been developed, reviewed, and approved by the ACMT Practice Committee and Board of Directors of the College and has been the subject of review and comment by members of the College. Disclosure statements for participating members of the ACMT Practice Committee and ACMT Board of Directors are available.

While the opinions of individual practitioners may differ, this is the position of the College at the time this document was written and approved after a thorough review of the issue and pertinent medical and scientific literature. 